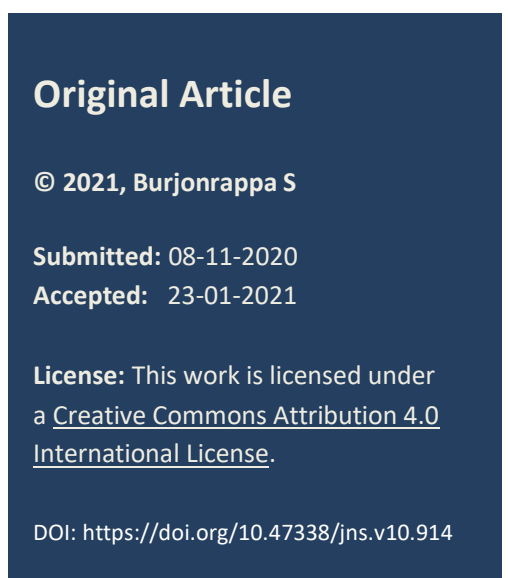

\title{
Long term pulmonary morbidity after esophageal atresia and tracheoesophageal fistula repair
}

\section{Sathyaprasad Burjonrappa}

Department of Surgery, Rutgers University, United States of America

Correspondence*: Sathyaprasad Burjonrappa, Department of Surgery, Rutgers University, United States of America E-mail: sathyabc@yahoo.com

\section{KEYWORDS}

Esophageal atresia, Pulmonary morbidity, GERD

ABSTRACT

Background: Respiratory morbidity can influence a patient's quality of life after successful EA/TEF (Esophageal Atresia with and without Tracheo-Esophageal Fistula) repair. Multidisciplinary clinics have made it easier to manage comorbidities in patients with complex congenital malformations. The aim of this study was to evaluate the impact of respiratory issues after EA/TEF repair. Secondarily we sought to evaluate the contribution of respiratory symptoms by Broncho-Pulmonary Dysplasia (BPD) and Gastro-EsophagealReflux-Disease (GERD) in patients with EA/TEF.

Methods: Retrospective review of the medical record of 50 patients, who underwent EA/TEF repair, needing hospital readmission for pulmonary morbidity, and subsequently followed up in a surgical clinic was performed. The data collected included patient demographics, presence and nature of significant respiratory comorbidity, findings on imaging studies and bronchoscopy, and results of pulmonary function tests (PFT).

Results: Respiratory issues were identified in $75 \%$ of the patients. Congenital malformations and tracheomalacia were found in $n=7(14 \%)$ of cases. Prematurity associated BPD and Gastro-Esophageal Reflux were not the major cause of respiratory symptoms. Respiratory morbidity in this population included recurrent pneumonia $n=18(36 \%)$, reactive airway disease $n=16(32 \%)$, bronchiolitis $n=4(8 \%)$, bronchiectasis $n=2(4 \%)$, laryngitis $n=2(4 \%)$ and empyema $n=1(2 \%)$.

Conclusions: Pulmonary complications significantly impact the quality of life in terms of respiratory events, after successful EA/TEF repair. While GERD is common in surgically repaired EA/TEF patients, its exact role in precipitating pulmonary morbidity needs further study. Tracheomalacia can be managed conservatively without resorting to aortopexy.
\end{abstract}

\section{INTRODUCTION}

Long term follow-up of neonates with complex congenital malformations is increasingly being recognized as an important aspect of pediatric surgical care.[1,2] This has led to the accumulation of a wealth of clinical data and has identified significant comorbidities affecting several body systems in these patients with complex malformations. This body of knowledge has helped develop protocols for follow-up of the asymptomatic individual and formulate screening methods and prophylactic intervention strategies for specific identified problems.[1-4] Respiratory infections, aspiration, and reactive airway disease are commonly seen in neonates undergoing surgical correction of $\mathrm{EA} / \mathrm{TEF}$. In this article, we aim to evaluate the burden of respiratory pathology and the incidence of res- piratory complications after EA/TEF repair. The primary objective was to determine the role of Broncho Pulmonary Dysplasia (BPD) and Gastro Esophageal Reflux Disease (GERD) in causing Respiratory Tract Infection (RTI). The secondary objective was to evaluate the burden of respiratory complications in this group and determine its impact on physician and hospital services

\section{METHODS}

After Institutional Review Board (IRB) approval, the medical records of all patients who had EA/TEF repair in the last 10 years with hospital readmission and subsequently followed up at a surgical clinic were retrospectively reviewed. Follow up after the initial discharge visit was at three monthly intervals for the first few years. Endoscopy and $\mathrm{pH}$ probe evaluation 
for reflux was performed if the child had symptomatic reflux. The frequency of visits to the clinic was subsequently tailored to the patients' need for evaluation. A standard respiratory health questionnaire was offered to all patients (or their caregivers) at each visit. This questionnaire evaluated the patient's respiratory symptoms; visit emergency rooms or to the primary care physician offices for respiratory problems, medication usage and frequency, and hospital admissions for respiratory decompensation in the interval between visits. All patients had a detailed physical examination performed and investigations were ordered based on examination findings. Radiological tests that were ordered included chest X-rays to evaluate pulmonary parenchymal abnormalities; cine-swallow studies to evaluate the deglutition defects and possible aspiration of upper digestive tract secretions and contents; upper gastrointestinal contrast studies to evaluate upper pouch anatomy and the anastomosis when compression from a dilated upper pouch was suspected to be exacerbating tracheomalacia, and rarely more advanced cross-sectional imaging studies (CT or MRI) were requested. Sputum analysis for evidence of invasive infection and identification of the organism and its antibiotic susceptibility was ordered when the patient could offer an adequate sample. Pulmonary Function Tests (PFT) were obtained after the age of seven years when the patients were capable of comprehending physician orders, cooperating with testing personnel, and coordinating their breathing patterns effectively. Forced vital capacity (FVC) and forced expiratory volume in one second (FEV1), as well as their reference values, were recorded. Results of PFT were classified as obstructive if $\mathrm{FEV} 1<80 \%$ (corresponding to a $z$-score-2.0) or restrictive if FEV1/FVC was $<85 \%$ (corresponding to a $z$-score2.0). Invasive tests such as bronchoscopy, BronchioAlveolar Lavage (BAL), and analysis for lipid-laden macrophages in the lavage fluid (indicative of aspiration) were performed if non-invasive tests suggested aspiration to be the cause for symptoms or if patients continued to have recurrent pneumonia despite adequate antibiotic prophylaxis. An experienced pathologist calculated LLMI (Lipid Laden Macrophage Index) after Oil Red-O fat staining of the BAL specimen. An LLMI greater than 200 was considered to represent a positive diagnostic test for aspiration. Evaluation and testing were coordinated with other specialists such as pediatric pulmonologists, occupational and physical therapists, gastroenterologists, and dietitians as needed.

Aspiration symptoms were addressed by long-term antibiotic treatment or by anti-reflux treatment in cases where GERD was thought to be the causative factor. Non-complicated RTI was treated with antibiotics, humidifiers, and bronchodilators. Established lower RTI were treated with intravenous antibiotics, inhalers, postural drainage, and physiotherapy on an inpatient basis. Flutter valves were used as modified positive expiratory pressure devices to vibrate the airways and facilitate the movement of mucus; to increase endobronchial pressure to avoid air trapping and to accelerate expiratory airflow to facilitate the upward movement of mucus. Bronchospasm and reactive airway disease were treated using betaagonists, steroid inhalers, leukotriene antagonists, or combinations of $\beta$ 2agonists and steroid inhalers. Parents were advised to administer prophylactic inhalers if they suspected a developing RTI, to minimize its consequences.

Tracheomalacia was treated conservatively in the majority. If symptoms failed to improve on conservative treatment and significant compression on the trachea was demonstrated on bronchoscopy, aortopexy was performed.

Data collection included demographics, gestational age, type of EA/TEF (Waterston classification), diagnosis of BPD, respiratory symptoms (wheeze, cough, sputum, shortness of breath), presence of GERD, respiratory medication use, the incidence of RTI, and Brief Resolved Unexplained Event (BRUE), and need for hospital admissions. BRUE was defined as the occurrence of one or more of the following: an episode of cyanosis or pallor; abnormal breathing pattern; change in tone; or altered responsiveness. Statistical analysis was performed using the Fisher test for categorical data and the student t-test for continuous variables. A p-value of $<0.05$ was considered significant.

\section{RESULTS}

Respiratory symptoms were identified in 39 of 50 $(78 \%)$ of the patients followed for a period of at least three years, while 11 never had a single respiratory event. While the mean gestational age of the entire study population was 37 weeks (range: 31-41 weeks), the mean gestational age of those with respiratory problems was 36 weeks and of those without was 37 weeks $(p=0.59)$. Wheezing and dry cough were seen in one-third of the patients after EA/TEF repair (Table I). There was no difference in the incidence of respiratory events in those with Type C (35/44) and those with Type A (3/5) fistulas $(p=0.31)$. GERD was diagnosed in $36(72 \%)$ of patients who had undergone EA/TEF repair after endoscopy and $\mathrm{pH}$ probe testing. Respiratory symptoms were seen in 30 of 36 patients (83\%) with proven reflux and 9 of 14 patients (64\%) without reflux $(p=0.2)$. There was no statistical difference in the prevalence of respiratory symptoms in the group that underwent surgical correction of reflux (13 of 17) as compared to the group that was treated medically (17 of 19) $(\mathrm{p}=0.2)$. Congenital malformations causing respiratory morbidity were seen in 7 (14\%) and included 1 pulmonary venous malformation, 1 pulmonary agenesis, 4 tracheomalacia, and 1 unilateral diaphragmatic paralysis. 
Table I: Illustrates the frequency of common respiratory symptoms in patients with EA/TEF

\begin{tabular}{|l|c|}
\hline Symptom & $\mathbf{N}(\%)$ \\
\hline Cough & $14(28 \%)$ \\
\hline Wheezing & $16(32 \%)$ \\
\hline Sputum & $4(8 \%)$ \\
\hline Stridor & $2(4 \%)$ \\
\hline Significant Respiratory Infections & $15(30 \%)$ \\
\hline Shortness of Breath & $5(10 \%)$ \\
\hline Laryngitis & $2(4 \%)$ \\
\hline Aspiration Pneumonia & $3(6 \%)$ \\
\hline Nasal congestion & $2(4 \%)$ \\
\hline BRUE & $1(2 \%)$ \\
\hline
\end{tabular}

Eight patients were able to produce reproducible PFT of which $6 / 8$ showed evidence of obstructive airway disease. The mean FEV in the tested patient subset was $71 \%$ (range: $38-87 \%$ ). The other two had normal PFT. Fifteen (30\%) patients developed respiratory in- fections needing a hospital admission in the study period. The lipid-laden macrophage index (LLMI) was elevated in three of eight patients with recurrent pneumonia revealing aspiration to be a cause in them. [5,6] Reactive airway disease was seen in 10 patients $(20 \%)$. Nine patients were diagnosed with asthma (18\%). Seventeen patients $(33 \%)$ were actively using bronchodilators and/or inhaled steroids. Leukotriene inhibitor was being used in three patients. In our experience, eight of the 39 patients (20\%) with a median age of 7 years (range 3.4-18.6 years) had complete resolution of symptoms. Brief Resolved Unexplained Event (BRUE) was identified in one patient only. All patients with tracheo-bronchomalacia were managed conservatively without an aortopexy. In this study, prematurity and its associated bronchopulmonary dysplasia were not significant factors in causing pulmonary morbidity. Table 2 summarizes findings in the study population.

Table 2: Table summarizing clinical findings in this population. (PFT: Pulmonary Function Test; LLM: Lipid Laden Macrophages; BRUE: Brief Resolved Unexplained Event)

\begin{tabular}{|l|l|l|}
\hline Clinical Finding & $\mathbf{N}(\%)$ & Finding/Comment \\
\hline PFT & $8(16 \%)$ & $\begin{array}{l}\text { 6/8 showed obstructive airway disease. (Mean FEV1 was 71\% } \\
(38-87 \%) \text { predicted) }\end{array}$ \\
\hline Respiratory Infections & $15(30 \%)$ & $\begin{array}{l}\text { LLM elevated in 3/8 patients with recurrent pneumonia indicat- } \\
\text { ing aspiration. }\end{array}$ \\
\hline RAD & $10(20 \%)$ & 9 diagnosed with Asthma \\
\hline Medication use & $\begin{array}{l}\text { Bronchodilator/Steroids } 17(34 \%) \\
\text { Leukotriene Inhibitor } 3(6 \%)\end{array}$ & $\begin{array}{l}\text { Airway reactivity is seen in a significant number after EA/TEF } \\
\text { repair }\end{array}$ \\
\hline BRUE & $1(2 \%)$ & Rare \\
\hline Need for Aortopexy & 0 & Not necessary in majority \\
\hline Complete resolution of symptoms & $8 / 39(21 \%)$ & At median age of 7 (3.4-18.6 years) \\
\hline
\end{tabular}

\section{DISCUSSION}

The prevalence of respiratory morbidity is very high in this group of patients and is akin to the experience of other large studies.[7-9] They are further prone to respiratory tract infections, reactive airway disease, and aspiration. Congenital malformations also influence their clinical course causing symptomatic dyspnea over the long term. A recent study from Finland showed a high incidence of restrictive lung disease and bronchial hyper-reactivity causing morbidity in adults who had undergone repair of EA/TEF. The results in this report were more like the results from the Finnish group reported in the adolescent population where about $30 \%$ had an obstructive spirometry defect. They attributed the restrictive lung disease to GERD and abnormal rib fusion secondary to the thoracotomy repair of the EA/TEF. They did not find airway inflammation to be significant in their studies with exhaled nitric oxide (FeNO).[7] PFTs done in patients in this study showed that an obstructive airway disease was more likely. In the pediatric age patients that did undergo PFTs in this study, obstructive airway disease was more likely as compared to the Finnish study that was carried out in adults. Calcification of the costal cartilages and decreasing chest wall compliance seems to increase the incidence of restrictive lung defects in the older population. There was a very high incidence of GERD after EA/TEF repair and a significant proportion of these patients had respiratory symptoms. GERD is often thought to precipitate respiratory crises in EA/TEF patients and is an indication for surgical correction of reflux.[10] However, the incidence of respiratory complications was no different in those with and without proven GERD. While not debating that some patients with recurrent aspiration and reactive airway disease do benefit from surgical correction of GERD, the incidence of respiratory problems was not significantly different in those who underwent surgical correction of GERD as compared to those who did not. With the caveat that the numbers in this study are small and susceptible to statistical error, further studies are needed to define the role of GERD in causing respiratory complications. The spectrum of GERD and the indications for antireflux surgery after EA/TEF repair may be answered with larger studies in the future. 
In this experience, while some babies were born premature, there was no significant difference in the incidence of prematurity between those with and those without respiratory issues. Other authors have also reported a similar experience.[11,12] Hence if one assumes prematurity as a surrogate for BPD the latter is not a major cause of pulmonary complications in this subset of patients. Surgical literature focuses often on the prevalence of tracheomalacia in patients after EA/TEF correction and its amelioration by aortopexy. [13] None of the patients in our series needed an aortopexy. It is best used sparingly and only in the most severe cases with expiratory tracheal collapse. The pathogenesis of BRUE in infants with esophageal atresia has been ascribed to GERD, tracheal compression between a dilated esophagus and an anomalous vessel [14], and/or weakness of the tracheal wall. [15] However recent studies seem to indicate that the etiology is multifactorial and the fundamental factor is likely a developmental delay of respiratory control. [16] Regular follow-up and treatment of reflux and prevention of respiratory infections by prophylactic intervention in a multi-disciplinary clinic seem to diminish the incidence of BRUE. We see these patients once every year in regular follow up until transition to

\section{REFERENCES}

1. Rasheed A, Tindall S, Cueny DL, Klein MD, DelaneyBlack V. Neurodevelopmental outcome after congenital diaphragmatic hernia: Extracorporeal membrane oxygenation before and after surgery. J Pediatr Surg. 2001; 36:539-44

2. Muratore CS, Kharasch V, Lund DP, Sheils C, Friedman $\mathrm{S}$, Utter S, et al. Pulmonary morbidity in 100 survivors of congenital diaphragmatic hernia monitored in a multidisciplinary clinic. J Pediatr Surg. 2001; 36:133-40.

3. Jancelewicz T, Vu LT, Keller RL, Bratton B, Lee H, Farmer D, et al. Long-term surgical outcomes in congenital diaphragmatic hernia: observations from a single institution. J Pediatr Surg. 2010; 45:155-60.

4. Holland AJ, Ron O, Pierro A, Drake D, Curry JI, Kiely EM, et al. Surgical outcomes of esophageal atresia without fistula for 24 years at a single institution. J Pediatr Surg. 2009; 44:1928-32.

5. Gratadour P, Védrinne JM, Guillaume C, Gagnieu MC, Motin J. Intra-macrophage lipid particles collected by bronchoalveolar lavage: incidence and diagnostic value. Ann Fr Anesth Reanim. 1993; 12:462-68.

6. Adams R, Ruffin R, Campbell D. The value of the lipidladen macrophage index in the assessment of aspiration pneumonia. Aust N Z J Med. 1997; 27:550-3.

7. Sistonen S, Malmberg P, Malmstrom K, Haahtela T, Sarna S, Rintala RJ, et al. Repaired esophageal atresia: Respiratory morbidity and pulmonary function in adults in a population-based long-term follow-up study. Eur Respir J. 2010; 36:1106-12.

8. Malmstrom K, Lohi J, Lindahl H, Pelkonen A, Kajosaari M, Sarna S, et al. Longitudinal follow-up of bronchial inflammation, respiratory symptoms, and pulmonary function in adolescents after repair of esophageal atre- adult clinics. However, all patients are seen sooner in case of aggravated reactive airway disease or after hospital admission for infection. While the overall respiratory status of a small proportion of these patients seems to improve with time, the majority remain symptomatic to varying degrees.

It is important to emphasize that in complex congenital malformations, long term follow-up is imperative, and such follow up should be led by the surgical team. Respiratory morbidity is seen in a majority after $\mathrm{EA} / \mathrm{TEF}$ repair. Some improvement in symptoms and exacerbations can be expected, as the child grows older. Significant proportions of EA/TEF patients have Reactive Airway Disease (RAD) and are dependent on medications for symptom control. Further studies are needed to evaluate the role of GERD in causing pulmonary morbidity.

Acknowledgements: Nil

Conflict of Interest: Authors have no conflict of interest. Source of Support: Nil

Consent to Publication: No clinical figure is used in this manuscript.

Author Contributions: Author(s) declared to fulfil authorship criteria as devised by ICMJE and approved the final version.

sia with tracheo esophageal fistula. J Pediatr. 2008; 46:396-401.

9. Patria MF, Ghislanzoni S, Macchini F, Lelii M, Mori A, Leva $\mathrm{E}$, et al. Respiratory morbidity in children with repaired congenital esophageal atresia with or without tracheoesophageal fistula. Int $\mathrm{J}$ Environ Res Public Health. 2017; 14:1136.

10. Rothenberg S, Cowles R. The effect of laparoscopic Nissen fundoplication on patients with severe gastroesophageal reflux disease and steroid dependent asthma. J Pediatr Surg. 2012; 47:1101-4.

11. Fayon M, Donato L. Tracheomalacia (TM) or bronchomalacia (BM) in children: conservative or invasive therapy. Arch Pediatr. 2010; 17:97-104.

12. Duerloo JA, Ekkelkamp S, Schoorl M, Heij HA, Aronson DC. Esophageal atresia: historical evolution of management and results in 371 patients. Ann Thorac Surg. 2002; 73:267-72.

13. Perger L, Kim HB, Jaksic T, Jennings RW, Linden BC. Thoracoscopic aortopexy for treatment of tracheomalacia in infants and children. J Laparoendosc Adv Surg Tech A. 2009; 19:S249-54.

14. Etesami M, Ashwath R, Kanne J, Gilkeson RC, Rajiah P. Computed tomography in the evaluation of vascular rings and slings. Insights Imaging. 2014; 5:507-21.

15. Sadreameli SC, McGrath-Morrow SA. Respiratory care of infants and children with tracheo-esophageal fistula and esophageal atresia. Pediatr Respir Rev. 2016; 17:16-23.

16. Cozzi DA, Zani A, Conforti A, Colarizi P, Moretti C, Cozzi F. Pathogenesis of apparent life-threatening events in infants with esophageal atresia. Pediatr Pulmonol. 2006; 41:488-93. 